\title{
Oligodramnia sem rotura das membranas amnióticas: resultados perinatais
}

\author{
Oligohydramnios without premature rupture of membranes: perinatal outcomes
}

José Mauro Madi ${ }^{1}$, Edson Nunes de Morais ${ }^{1,2}$, Breno Fauth de Araújo ${ }^{1}$, Renato Luís Rombaldi ${ }^{1}$, Sônia Regina Cabral Madi ${ }^{1}$, Luciano Ártico ${ }^{1}$, Natacha Araújo Machado ${ }^{1}$

\section{RESUMO}

Objetivo: avaliar os resultados perinatais em casos de oligodramnia sem rotura de membranas amnióticas. Métodos: foram estudados retrospectivamente 51 casos consecutivos de oligodramnia (índice de líquido amniótico (ILA) menor que $5 \mathrm{~cm}$ ) em nascimentos ocorridos no período de março de 1998 a setembro de 2001. Compararam-se os dados obtidos aos de 61 casos com quantidade intermediária e normal de líquido amniótico (ILA $>5 \mathrm{~cm}$ ). Analisaram-se variáveis maternas e neonatais, bem como taxas de mortalidade fetal, neonatal precoce e perinatal. As avaliações estatísticas foram realizadas mediante a aplicação do teste não paramétrico do $\chi^{2}$ com a correção de Yates, e do teste $t$ de Student. Adotou-se o nível de significância de 5\%. Resultados: não houve diferença significante entre os grupos estudados, ao se analisar a ocorrência de síndrome hipertensiva, presença de mecônio, índice de Apgar inferior a sete no primeiro e quinto minuto, internação na unidade de tratamento intensivo neonatal e prematuridade. A oligodramnia associou-se significantemente ao tipo de parto $(p<0,0002$; $R R=0,32)$, sofrimento fetal agudo ( $<<0,0004 ; R R=2,2)$ e presença de malformações fetais $(p<0,01 ; R R=5,4)$. Os percentuais de malformações fetais foram de 17,6 e 3,3\% nos grupos de oligodramnia e normal, respectivamente. As taxas de mortalidade fetal $(2,0 v s$ 1,6\%), neonatal $(5,9 v s$ 1,6\%) e perinatal (7,8 vs 3,3\%), em ambos os grupos, não apresentaram diferença significante. Conclusão: a oligodramnia se associou a um aumento do risco para operação cesariana, sofrimento fetal agudo e malformações fetais.

PALAVRAS-CHAVE: Morbidade perinatal; Mortalidade perinatal; Malformações fetais; Oligodramnia

\section{ABSTRACT}

Purpose: to evaluate perinatal outcomes in cases of oligohydramnios without premature rupture of membranes. Methods: a total of 51 consecutive cases of oligohydramnios (amniotic fluid index, AFI $<5 \mathrm{~cm}$ ) born between March 1998 and September 2001 were studied retrospectively. Data were compared to 61 cases with intermediate and normal volume of amniotic fluid AFI $>5$ ). Maternal and neonatal variables, as well as fetal mortality, early neonatal, and perinatal mortality rates were analyzed. For statistical analysis the $\chi^{2}$ test with Yates correction and Student's $t$ test were used with level of signicance set at $5 \%$. Results: there were no significant differences between groups when the presence of gestational hypertensive syndromes, meconium-stained amniotic fluid, 1- and 5-minute Apgar score, need of neonatal intensive center unit, and preterm birth were analyzed. Oligohydramnios was associated with the way of delivery $(\mathrm{p}<0.0002 ; \mathrm{RR}=0.3)$, fetal distress $(p<0.0004 ; R R=2.2)$ and fetal malformations $(p<0.01 ; R R=5.4)$. Fetal malformation rates were 17.6 and $3.3 \%$ in oligohydramnios and normal groups, respectively. Fetal mortality ( $2.0 \mathrm{vs} 1.6 \%)$, early neonatal (5.9 vs $1.6 \%)$ and perinatal mortality (7.8 vs $3.3 \%$ ) rates in both groups did not show statistical significance. Conclusion: Oligohydramnios was related to increased risk factor for cesarean section, fetal distress and fetal malformations.

KEYWORDS: Perinatal morbidity; Perinatal mortality; Fetal malformation; Oligohydramnios

\footnotetext{
1 Centro de Ciências Biológicas e da Saúde da Universidade de Caxias do Sul

2 Universidade Federal de Santa Maria

Correspondência: José Mauro Madi

Serviço de Obstetrícia do Hospital Geral de Caxias do Sul

Rua Antonio Vignolli, 255 - Bairro Petrópolis - 95001-970 - Caxias do Sul - RS - Fone/Fax (54) 229-4444 - e-mail: madi@visao.com.br

Recebido em: 9/8/2004 Aceito com modificações em: 7/2/2005
} 
Introdução

Oligodramnia é a diminuição do volume de líquido amniótico para uma idade gestacional definida. Sua incidência varia de 3,9 a 5,5\% de todas gestações ${ }^{1-3}$. As principais causas determinantes da diminuição do volume âmnico são a ruptura prematura das membranas amnióticas ${ }^{3,4}$, a insuficiência placentária, a presença de anomalias fetais (mormente as do sistema urinário) $)^{1,5}$, as sindromes hipertensivas $^{6-8}$, o tabagismo ${ }^{9}$ e a pós-maturidade ${ }^{10,11}$.

Diante de uma situação de hipoxia crônica, o feto procura adaptar-se à nova condição imposta pelo meio onde se encontra, protegendo órgãos nobres com sacrificio de outros menos significativos, por meio da redistribuição do fluxo sangüineo (fenômeno da centralização). Assim, as coronárias e as carótidas são beneficiadas com maior fluxo oriundo do débito cardíaco; em oposição, os pulmões e os rins são privados de fluxo sangüíneo ideal, culminando em oligodramnia pela diminuição da diurese e da secreção de fluidos pulmonares.

A oligodramnia é suspeitada pelo exame fisi$\mathrm{Co}^{12}$, quando de medidas da altura uterina inferiores para a idade gestacional, e confirmada por exame ultra-sonográfico, que fornece o diagnóstico de acordo com o índice de líquido amniótico (ILA) ${ }^{6,13}$.

A oligodramnia associa-se ao crescimento intra-uterino restrito ${ }^{13,14}$, taxas mais elevadas de nascimentos pela via abdominal, baixos índices de Apgar no quinto minuto, mortalidade perinatal $^{13,14}$, presença de mecônio no líquido amniótico e sofrimento fetal, seja devido à doença de base que o determinou, seja em decorrência da compressão exercida pela parede uterina diretamente sobre o corpo do concepto.

O objetivo do presente trabalho foi o de estudar casos de oligoidramnia sem ruptura da membrana amniótica, ocorridos em uma população de um hospital-escola, sob o ponto de vista obstétrico e neonatal.

\section{Pacientes e Métodos}

Realizou-se estudo transversal, no qual foram incluídos 6.060 prontuários de pacientes que foram atendidas consecutivamente na Unidade de Medicina Perinatal do Hospital Geral da Universidade de Caxias do Sul, no período de março de 1998 a setembro de 2001, com a finalidade de se identificarem aquelas que apresentaram diagnóstico ultra-sonográfico de oligodramnia.

De um total de 105 casos de oligodramnia, foram selecionados 51 prontuários de gestantes sem ruptura de membranas amnióticas como fator etiológico, e excluídos 54 casos, por não apre- sentarem na íntegra os dados necessários à pesquisa. Para fins de análise, compararam-se os dados obtidos a 61 casos com quantidade intermediária e normal de líquido amniótico (ILA $>5 \mathrm{~cm}$ ), selecionados de acordo com a integridade dos dados clínicos e ocorridos imediatamente após o desfecho dos casos que cursaram com oligodramnia. Os critérios de seleção da amostra para ambos os grupos foram: parto e diagnóstico ultra-sonográfico de oligodramnia realizados na instituição e dados clínicos anotados com clareza e exatidão.

Considerou-se como diminuição do líquido amniótico a presença de ILA inferior a $5 \mathrm{~cm}$, mensurado segundo a técnica descrita por Phelan et al. ${ }^{15}$ (técnica dos quatro quadrantes), que consiste em se dividir o abdômen materno em quatro quadrantes, utilizando-se a linha nigrans como eixo longitudinal e a cicatriz umbilical como referência para o eixo transverso. É, então, medido o diâmetro vertical do maior bolsão de líquido amniótico de cada quadrante e a soma dos valores dos quatro bolsões constitui o ILA, que pode ser assim classificado: oligodramnia, inferior a $5 \mathrm{~cm}$; intermediário, de $5 \mathrm{~cm}$ a $8 \mathrm{~cm}$; normal, de $8 \mathrm{~cm}$ a $18 \mathrm{~cm}$, e polidramnia, superior a $18 \mathrm{~cm}^{15}$.

Analisaram-se as seguintes variáveis: idade gestacional (em semanas) ocorrência de hipertensão prévia à atual gestação, a via de parto, a presença de qualquer quantidade de mecônio no líquido amniótico, índice de Apgar inferior a sete no primeiro e quinto minutos, peso do recém-nascido, necessidade de internação em ambiente de intensivismo e as ocorrências de prematuridade (tempo de gravidez inferior a 37 semanas), síndrome de aspiração do mecônio, todos os tipos de malformações fetais, crescimento intra-uterino restrito e sofrimento fetal agudo, bem como as taxas de mortalidade fetal (natimortalidade), neonatal precoce (até sete dias) e perinatal.

Considerou-se como sindrome de aspiração de mecônio a presença de mecônio nas cordas vocais do recém-nascido, por ocasião do nascimento, além dos sinais clínicos respiratórios e radiológicos observados durante a internação na unidade de tratamento intensivo.

O diagnóstico de sofrimento fetal foi realizado quando da presença de alterações da freqüência cardíaca fetal no registro cardiotocográfico, segundo os critérios do National Institutes of Health $(1997)^{16}$, associadas à presença de mecônio no líquido amniótico. Considerou-se crescimento intrauterino restrito, o peso do recém-nascido abaixo do $10^{\circ}$ percentil, segundo Battaglia e Lubchenko ${ }^{17}$.

A doença da membrana hialina foi diagnosticada pelo início da disfunção respiratória nas primeiras horas de vida, com progressão por 48 a 72 horas. A comprovação ocorreu mediante raios $\mathrm{X}$ de tórax.

Os dados obstétricos e perinatais foram inseridos no SPSSPC 10.0 (Statistical Package for the Soci- 
al Sciences for Personal Computer) e as avaliações estatísticas foram realizadas mediante a aplicação do teste não paramétrico do $\chi^{2}$ com a correção de Yates (via de parto, necessidade de cuidados de intensivismo neonatal e ocorrência de hipertensão prévia à atual gestação, de mecônio no líquido amniótico, de prematuridade, de malformações fetais e de sofrimento fetal agudo) e do teste $t$ de Student (peso do recém-nascido, idade gestacional e índice de Apgar inferior a sete no primeiro e quinto minutos). Adotou-se o nível de significância de 5\%.

O projeto de pesquisa foi aprovado pela Comissão de Ética em Pesquisa da Universidade de Caxias do Sul.

\section{Resultados}

A idade gestacional média por ocasião da interrupção da gestação foi de 39 semanas para ambos os grupos, não havendo, portanto, diferença significante entre eles. O peso médio dos recém-nascidos do grupo portador de oligodramnia foi de 2.700 vs. $3.185 \mathrm{~g}$, observado na amostra comparativa.

Não houve diferença significante entre os grupos analisados ao se compararem a ocorrência de hipertensão materna prévia à gestação, presença de mecônio no líquido amniótico por ocasião do parto, indice de Apgar inferior a sete no primeiro e quinto minuto, necessidade de internação em unidade de tratamento intensivo neonatal e prematuridade (Tabela 1). A análise dos casos com índice normal de líquido amniótico e oligodramnia não identificou a ocorrência de sindrome de aspiração de mecônio.

A oligodramnia associou-se significantemente à via de parto $(\mathrm{RR}=0,3$ e $\mathrm{OR}=0,1$; IC $95 \%$ : $0,04-0,3 ; p<0,0002)$. O índice de partos cesáreos no grupo que cursou com oligodramnia $(n=36$; $70,6 \%$ ) foi três vezes superior ao grupo de pacientes que cursou com ILA normal ( $n=13 ; 21,3 \%)$. A diminuição do volume de líquido amniótico associou-se significantemente ao sofrimento fetal agudo (RR=2,2 e OR=8, 1 ; IC 95\%: 2,0-37,9; $p<0,0004)$, observando-se taxa cinco vezes superior de comprometimento fetal no grupo de oligodramnia.

Casos de crescimento intra-uterino restrito só foram observados na presença de oligodramnia ( $n=9 ; 17,6 \%)$. As taxas de mortalidade fetal, neonatal e perinatal podem ser vistas na Tabela 2, não sendo observada associação significante entre as taxas citadas e a diminuição do líquido amniótico.

Malformações fetais $(\mathrm{RR}=5,4$ e $\mathrm{OR}=6,3$; IC 95\%: $1,2-44,8 ; \mathrm{p}<0,01)$ foram cerca de cinco vezes mais freqüentes entre os casos de diminuição do volume de líquido amniótico (Tabela 3). Foram identificados cinco casos de malformação do apa- relho geniturinário ( $\mathrm{n}=4 ; 44,4 \%$ vs $\mathrm{n}=1 ; 50 \%)$, dois casos de sistema nervoso central $(\mathrm{n}=1 ; 11,2 \%$ vs $\mathrm{n}=1 ; 50 \%$ ) e quatro casos de associação de malformações ( $\mathrm{n}=4 ; 44,4 \%$ vs $\mathrm{n}=0)$ nos grupos de oligodramnia e líquido amniótico normal, respectivamente. As malformações do sistema geniturinário prevaleceram sobre as demais.

\section{Discussão}

O líquido amniótico é componente importante do ambiente intra-uterino. Sua produção e a sua absorção dependem de uma série de mecanismos interdependentes entre o feto, a placenta, as membranas e o organismo materno, sendo que qualquer alteração no seu volume requer cuidadosas avaliações tanto do feto como da mãe ${ }^{13}$.

Tabela 1 - Variáveis obstétricas e perinatais nos grupos de pacientes com oligodramnia (índice de líquido amniótico) <5) e com volume de líquido amniótico normal.

\begin{tabular}{|c|c|c|c|c|c|}
\hline \multirow[t]{4}{*}{ Variáveis } & \multicolumn{4}{|c|}{ Índice de líquido amniótico $(\mathrm{cm})$} & \multirow[t]{4}{*}{$p$} \\
\hline & & & \multirow{2}{*}{\multicolumn{2}{|c|}{$n=61$}} & \\
\hline & \multicolumn{2}{|c|}{$\mathrm{n}=51$} & & & \\
\hline & $\mathrm{n}$ & $\%$ & $\mathrm{n}$ & $\%$ & \\
\hline HAS & 2 & 3,9 & 5 & 8,2 & NS \\
\hline Mecônio & 6 & 11,8 & 9 & 14,8 & \\
\hline Apgar de $1^{\circ}$ minuto $<7$ & 10 & 19,6 & 11 & 18,0 & NS \\
\hline Apgar de $5^{\circ}$ minuto $<7$ & 5 & 9,8 & 2 & 3,3 & NS \\
\hline Internação na UTIN & 16 & 31,4 & 14 & 23,0 & NS \\
\hline Prematuridade & 6 & 11,8 & 3 & 4,9 & NS \\
\hline
\end{tabular}

HAS: hipertensão prévia à gestação atual; UTIN: unidade de tratamento intensivo neonatal.

Tabela 2 - Distribuição das taxas de mortalidade fetal, neonatal e perinatal.

\begin{tabular}{|c|c|c|c|c|c|}
\hline & \multicolumn{4}{|c|}{ Índice de líquido amniótico (cm) } & \multirow[t]{4}{*}{$p$} \\
\hline & \multirow{2}{*}{\multicolumn{2}{|c|}{$\begin{array}{c}<5 \\
n=51\end{array}$}} & \multirow{2}{*}{\multicolumn{2}{|c|}{$\begin{array}{c}>5 \\
n=61\end{array}$}} & \\
\hline & & & & & \\
\hline & $\mathrm{n}$ & $\%$ & $\mathrm{n}$ & $\%$ & \\
\hline TMF & 1 & 2,0 & 1 & 1,6 & NS \\
\hline TMN & 3 & 5,9 & 1 & 1,6 & NS \\
\hline TMP & 4 & 7,8 & 2 & 3,3 & NS \\
\hline
\end{tabular}

TMF: taxa de mortalidade fetal; TMN: taxa de mortalidade neonatal; TMP: taxa de mortalidade perinatal.

Tabela 3 - Estudo das variáveis obstétricas e perinatais. Comparação entre grupos com índice de líquido amniótico menor que 5 e maior que 5 .

\begin{tabular}{ccc}
\hline Índice de líquido amniótico & $(\mathrm{cm})$ & $\mathrm{p}$ \\
$<5$ & $>5$ & \\
$n=51$ & $n=61$ & \\
$n \quad \%$ & $n \quad \%$ &
\end{tabular}

\begin{tabular}{|c|c|c|c|c|c|}
\hline \multicolumn{6}{|l|}{ Tipo de parto } \\
\hline Vaginal & 15 & 29,4 & 48 & 78,7 & \\
\hline Cesáreo & 36 & 70,6 & 13 & 21,3 & $<0,0002^{*}$ \\
\hline SFA & 15 & 29,4 & 3 & 4,9 & $<0,0004^{* *}$ \\
\hline Malformação congênita & 9 & 17,6 & 2 & 3,3 & $<0,01^{* * *}$ \\
\hline
\end{tabular}


Assim, o estudo do ILA é uma das melhores maneiras de se avaliarem os resultados perinatais associados às alterações de volume do fluido âmnico. Para levá-lo a cabo há que se trabalhar com equipe afeita aos meandros da Medicina Perinatal e a critérios padronizados que propiciem seguimento e decisões adequadas ${ }^{15,18}$.

$\mathrm{O}$ estudo em questão identificou diferença significante na análise das variáveis tipo de parto, sofrimento fetal agudo e ocorrência de malformações fetais; por outro lado, as variáveis ocorrência de síndromes hipertensivas e mecônio no líquido amniótico, índice de Apgar inferior a sete no primeiro e quinto minuto, necessidade de internação em unidade de tratamento intensivo neonatal, prematuridade e taxas de mortalidade neonatal, fetal e perinatal não estiveram associadas ao ILA inferior a $5 \mathrm{~cm}$.

O crescimento intra-uterino restrito foi diagnosticado em $9(17,6 \%)$ dos casos de oligodramnia, estando ausente no grupo de gestantes que cursou com líquido amniótico normal. Alguns autores referem taxas de crescimento intra-uterino restrito de até $36,6 \%$ na presença de oligoidramnia ${ }^{19}$. Nomura et al. ${ }^{20}$ observou incidência de $21 \%$ numa população portadora de oligodramnia grave.

A fisiopatologia do crescimento intra-uterino restrito está intimamente relacionada à hipoxia crônica e à diminuição do fluxo renal e pulmonar fetal, originando diminuição do líquido amniótico e provocando um meio intra-uterino hostil ao concepto. É a única variável que se apresenta de maneira consensual na literatura compila$\mathrm{da}^{6,8,14,19}$. Banks e Millar ${ }^{21}$ relacionaram o oligodrâmnio moderado (ILA $>5 \mathrm{~cm} \mathrm{e}<10 \mathrm{~cm}$ ) ao crescimento intra-uterino restrito.

A ocorrência de sofrimento fetal agudo também foi significantemente maior no grupo portador de oligodramnia $(n=15 ; 29,4 \%)$ do que no grupo que cursou com ILA $>5 \mathrm{~cm}(\mathrm{n}=3 ; 4,9 \%)$, sendo o risco relativo de 2,2 e $p<0,0004$. O sofrimento fetal é decorrente da acidose metabólica induzida pela hipoxia vigente, tanto aguda quanto crônica. Esse evento pode ser comprovado pelas modificações da freqüência cardíaca fetal à cardiotocografia intraparto ${ }^{14,22}$ e pelo baixo $\mathrm{pH}$ observado na gasometria arterial do cordão umbilical ao nascimento $^{7}$. É aceita a hipótese de que a incidência e a gravidade do sofrimento fetal são proporcionais à compressão do cordão umbilical, que por sua vez pode ocasionar quadro de hipoxia fetal se a interferência sobre o fluxo sangüíneo funicular for severa, repetitiva e prolongada ${ }^{18}$.

A sindrome de aspiração do mecônio, a despeito das várias associações com a diminuição do líquido amniótico observadas na literatura, não foi identificada no estudo ${ }^{20,23}$. O tamanho amostral analisado provavelmente não foi suficiente para permitir a identificação dessa doença.

Em relação ao tipo de parto, identificou-se índice de $70,6 \%$ de cesáreas em pacientes do grupo de oligodramnia e $21,3 \%$ no grupo com ILA normal (OR=0,1 e RR=0,3; $p<0,0002)$. Os trabalhos consultados citam a operação cesariana como a via de parto preferida em casos de oligodramnia, coincidindo com os resultados encontrados nesta pesquisa $^{6,18,24}$. Os indices publicados variam de 60 a $66 \%$ em pacientes com oligodrâmnio vs. $32 \%$ de partos vaginais na população geral ${ }^{18,19}$. Observase, na prática, maior liberalidade de médico assistente em indicar a tomotocia. Some-se a esse fato, a ocorrência de sofrimento fetal $(n=15 ; 29,4$ vs $\mathrm{n}=3 ; 4,9 \%$ ) ocasionado pela compressão do cordão umbilical e diagnosticado pelo alterações da freqüência cardiaca fetal e/ou presença de mecônio no líquido amniótico, e pela crescimento intra-uterino restrito, caracterizado pela presença de insuficiência placentária $(\mathrm{n}=9 ; 17,6 \%$ vs $\mathrm{n}=0$ ), nos grupo de oligodramnia e normal, respectivamente. Um incremento nas taxas de parto operatório é a resultante dessas associações.

As sindromes hipertensivas, apontadas como fatores indutores de oligodrâmnio ${ }^{6}$, não apresentaram diferença estatisticamente significante entre os grupos estudados ( $n=2 ; 3,9$ vs $n=5 ; 8,2 \%)$. Esse resultado pode ser explicado pelo tamanho amostral e pela população não selecionada. Bastos et al. ${ }^{25}$, num estudo que incluiu 27 casos de oligodramnia, observaram 10 casos de hipertensão arterial (37\%).

Índices de Apgar inferior a sete no primeiro e no quinto minuto não estiveram associados ao ILA diminuído. Condutas mais agressivas, caracterizadas pela cesárea eletiva e pela não indução do trabalho de parto, podem ter agido como fator de proteção fetal ${ }^{6,15,18}$.

A presença de malformações fetais foram achados importantes $(n=9,17,6 \%$; OR=6,3, $R R=5,4$; $\mathrm{p}<0,01$ ), pois comprovaram que o oligodrâmnio pode advir de falhas na formação de segmentos corporais, podendo influenciar na formação da urina fetal. Sabe-se que as malformações renais são as mais importantes causas de oligodramnia, posto que, ao final da gestação, a urina fetal é o principal componente do liquido amniótico; assim, quando da agenesia renal, rim policístico ou qualquer outra patologia renal que interfira na produção de urina, diminuindo-a, a oligodramnia é a conseqüência mais destacada. As malformações cerebrais e as digestivas também estão associadas ao aparecimento da oligodramnia, já que os fetos portadores não conseguem deglutir o liquido amniótico, alterando a sua absorção pela via digestiva e a conseqüente eliminação urinária. Bastos et $a 1 .^{25}$, num estudo que incluiu 27 casos de oligodramnia sem ruptura da membrana amniótica em gestações inferiores a 26 semanas, observaram treze casos de malformação fetal, sendo oito do trato urinário $(29,6 \%)$ e quatro do sistema nervoso central $(14,8 \%)$. Cunha et al. ${ }^{26}$ referiram que a maioria das anomalias congênitas envolvidas com a oligodramnia 
acomete o trato geniturinário, devido, provavelmente, ao desequilíbrio entre a produção e a excreção da urina fetal.

A presença de mecônio no líquido amniótico, embora presente no grupo portador de oligodramnia e no grupo normal, 11,8 vs $14,8 \%$, respectivamente, encontra-se dentro da faixa tradicionalmente citada na literatura para a população geral. Na UMPHG-UCS, a taxa de mecônio por ocasião dos partos é de $15,5 \%{ }^{24}$. Nomura et al. ${ }^{20}$ observaram incidência de 16,8\% de líquido amniótico meconial.

A mortalidade fetal nesse estudo, associada ao ILA $<5 \mathrm{~cm}$, alcançou índice de 1,96 vs $1,64 \%$ (ILA $>5 \mathrm{~cm}$ ); esse dado coincide com o observado por outros autores que afirmam ser significante a sua relação com a oligodramnia, normalmente ao redor de $5 \%$ de gestações comprometidas e 0,3\% em gestações normais ${ }^{18,19}$. A explicação para o fato pode estar vinculada à presença concomitante de sofrimento fetal agudo e, principalmente, do crescimento intra-uterino restrito e das malformações fetais. A neomortalidade precoce e a perinatal não mostraram associação significante; refletem, via de regra, a boa atuação neonatal.

O estudo comprovou os efeitos deletérios da oligodramnia sobre a saúde fetal, ao demonstrar importante associação com a morbidade perinatal $^{8,19}$, e fazendo crer que o acompanhamento prénatal, nesses casos, deva ter freqüência e qualidade melhoradas em serviços especializados, quando disponíveis. Indubitavelmente, a diminuição do volume de líquido amniótico associa-se a prognóstico fetal sombrio e a resultados perinatais adversos.

\section{Referências}

1. Magann EF, Chauhan SP, Kinsella MJ, McNamara MF, Whitworth NS, Morrison JC. Antenatal testing among 1001 patients at high risk: the role of ultrasonographic estimate of amniotic fluid volume. Am J Obstet Gynecol. 1999; 180:1330-6.

2. Mercer LJ, Brown LG, Petres RE, Messer RH. A survey of pregnancies complicated by decreased amniotic fluid. Am J Obstet Gynecol. 1984; 149:355-61.

3. Philipson EH, Sokol RJ, Williams T. Oligohydramnios: clinical associations and predictive value for intrauterine growth retardation. Am J Obstet Gynecol. 1983; 146:271-8.

4. Park JS, Yoon BH, Romero R, et al. The relationship between oligohydramnios and the onset of preterm labor in preterm premature rupture of membranes. Am J Obstet Gynecol. 2001; 184:459-62.

5. Ghosh G, Marsal K, Gudmundsson S. Amniotic fluid index in low-risk pregnancy as an admission test to the labor ward. Acta Obstet Gynecol Scand. 2002; 81:852-5.

6. Chamberlain PF, Manning FA, Morrison I, Harman CR, Lange IR. Ultrasound evaluation of amniotic fluid volume II. The relationship of increased amniotic fluid volume to perinatal outcome. Am J Obstet Gynecol. 1984; 150:250-4.

7. Illia R, Mayer H, Marzik C, et al. Resultados perinatales asociados al tratamiento conservador del oligoamnios durante el embarazo. Toko-Ginecol Pract. 2001; 60:238-42.
8. Casey BM, McIntire DD, Bloom SL, et al. Pregnancy outcomes after antepartum diagnosis of oligohydramnios at or beyond 43 weeks' gestation. Am J Obstet Gynecol. 2000; 182:909-12.

9. Schucker JL, Mercer BM, Audibert F, Lewis RL, Friedman SA, Sibai BM. Serial amniotic fluid index in severe preeclampsia: a poor predictor of adverse outcome. Am J Obstet Gynecol. 1996; 175:1018-23.

10.Tarari S, Treisser A, Renaud R. Oligohydramnios. Diagnosis. Etiology. Prognosis. J Gynecol Obstet Biol Reprod (Paris). 1987; 16:755-63.

11.Clement D, Schifrin BS, Kates RB. Acute oligohydramnios in postdate pregnancy. Am J Obstet Gynecol. 1987; 157:884-6.

12. Crowley P. Non quantitative estimation of amniotic fluid volume in suspected prolonged pregnancy. J Perinat Med. 1980; 8:249-51.

13. Mauad Filho F, Molinari MB, Amaral VF. Oligoidramnia. In: Morais EN, Mauad Filho F, editores. Medicina Materna e Perinatal. $1^{a}$ ed. Rio de Janeiro: Revinter; 2000. p. 184-7.

14.Grubb DK, Paul RH. Amniotic fluid index and prolonged antepartum fetal heart rate decelerations. Obstet Gynecol. 1992; 79:558-60.

15.Phelan JP, Smith CV, Broussard R, Small M. Amniotic fluid volume assessment with the four- quadrant technique at 36-42 weeks' gestation. J Reprod Med. 1987; 32:540-2.

16.National Institutes of Health. Electronic fetal heart rate monitoring: research guidelines for interpretation. Am J Obstet Gynecol. 1997; 177:1385-90.

17. Battaglia FC, Lubchenko LO. A practical classification of newborn infants by weight and gestational age. J Pediatr. 1967; 71:159-63.

18.Voxman EG, Tran S, Wing DA. Low amniotic fluid index as a predictor of adverse perinatal outcome. J Perinatol. 2002; 22:282-5.

19.Rainford M, Adair R, Scialli AR, Ghidini A, Spong CY. Amniotic fluid index in the uncomplicated term pregnancy. Prediction of outcome. J Reprod Med. 2001; 46:589-92.

20.Nomura RMY, Francisco RPV, Miyadahira S, Zugaib M. Análise dos testes de vitalidade fetal e dos resultados perinatais em gestações de alto risco com oligodrâmnio. Rev Bras Ginecol Obstet. 2002; 24:401-6.

21.Banks EH, Millar DA. Perinatal risks associated with borderline amniotic fluid index. Am J Obstet Gynecol. 1999; 180:1461-3.

22.Magann EF, Kinsella MJ, Chauhan SP, McNamara MF, Gehring BW, Morison JC. Does an amniotic fluid index of d" $5 \mathrm{~cm}$ necessitate delivery in high-risk pregnancies? A case-control study. Am J Obstet Gynecol. 1999; 180:1354-9.

23.Madi JM, Morais EN, Locatelli E, Madi, SRC, Rombaldi RL. Síndrome de aspiração do mecônio: análise dos resultados obstétricos e perinatais. Rev Bras Ginecol Obstet. 2003; 25:123-8.

24. Chauhan SP, Sanderson M, Hendrix NW, Magann EF, Devoe LD. Perinatal outcome and amniotic fluid index in the antepartum and intrapartum periods: a metaanalysis. Am J Obstet Gynecol. 1999; 181:1473-8.

25.Bastos GZG, Nogueira RO, Alencar Júnior CA. Repercussões perinatais do oligoidrâmnio sem ruptura prematura das membranas detectado até a $26^{a}$ semana de gravidez. Rev Bras Ginecol Obstet. 2003; 25:419-23.

26. Cunha SP, Peraçoli JC, Duarte G, et al. Influência da hipertensão arterial durante a gravidez sobre os índices de Apgar no primeiro e quinto minutos. Rev Bras Ginecol Obstet. 1986; 8:18-21. 